\title{
The Iliocapsularis Muscle
}

\section{An Important Stabilizer in the Dysplastic Hip}

\author{
D. Babst MD, S. D. Steppacher MD, R. Ganz MD, \\ K. A. Siebenrock MD, M. Tannast MD
}

Received: 12 May 2010/Accepted: 15 November 2010/Published online: 3 December 2010

(C) The Association of Bone and Joint Surgeons ( 2010

\begin{abstract}
Background The iliocapsularis muscle is a little known muscle overlying the anterior hip capsule postulated to function as a stabilizer of dysplastic hips. Theoretically, this muscle would be hypertrophied in dysplastic hips and, conversely, atrophied in stable and well-constrained hips. However, these observations have not been confirmed and the true function of this muscle remains unknown.

Questions/purposes We quantified the anatomic dimensions and degree of fatty infiltration of the iliocapsularis muscle and compared the results for 45 hips with deficient acetabular coverage (Group I) with 40 hips with excessive acetabular coverage (Group II).

Patients and Methods We used MR arthrography to evaluate anatomic dimensions (thickness, width, circumference, cross-sectional area [CSA], and partial volume) and the amount of fatty infiltration.

Results We observed increased thickness, width, circumference, CSA, and partial volume of the iliocapsularis muscle in Group I when compared with Group II. Additionally, hips in Group I had a lower prevalence of fatty infiltration compared with those in Group II. The
\end{abstract}

Each author certifies that he or she has no commercial associations that might pose a conflict of interest in connection with the submitted article.

Each author certifies that his or her institution has approved the human protocol for this investigation and that all investigations were conducted in conformity with ethical principles of research.

This work was performed at the University of Bern, Switzerland

D. Babst, S. D. Steppacher ( $₫)$, R. Ganz,

K. A. Siebenrock, M. Tannast

Department of Orthopaedic Surgery, Inselspital, University

of Bern, Murtenstrasse, 3010 Bern, Switzerland

e-mail: simon.steppacher@insel.ch;

simon.steppacher@gmail.com iliocapsularis muscle typically was hypertrophied, and there was less fatty infiltration in dysplastic hips compared with hips with excessive acetabular coverage.

Conclusion These observations suggest the iliocapsularis muscle is important for stabilizing the femoral head in a deficient acetabulum. This muscle serves as an anatomic landmark when performing a periacetabular osteotomy. Additionally, preoperative evaluation of morphologic features of the muscle can be used as an adjunct for decision making when treating patients with borderline hip dysplasia or femoroacetabular impingement.

\section{Introduction}

The iliocapsularis muscle (also called iliacus minor or iliotrochantericus) is a little known, but uniformly present muscle overlying the anterior hip capsule (Fig. 1) [29]. It originates from the anteromedial hip capsule and anteriorinferior iliac spine (AIIS). Its insertion is located just distal to the lesser trochanter (Fig. 2) [29]. Although the true function of the iliocapsularis muscle is unknown, it has been suggested the iliocapsularis muscle tightens the hip capsule [1, 26, 29]. Theoretically, this is particularly important in dysplastic hips, where this muscle contraction would help stabilize the femoral head in the deficient acetabulum. If this is correct, then one would anticipate the muscle would be hypertrophied in dysplastic hips and, conversely, atrophied in stable and well-constrained hips with excessive acetabular coverage. Supporting this theory, Ward et al. [29] observed a more prominent iliocapsularis muscle when performing periacetabular osteotomy for hips with dysplasia compared with surgery in nondysplastic hips (acetabular and femoral head fractures). However, these 


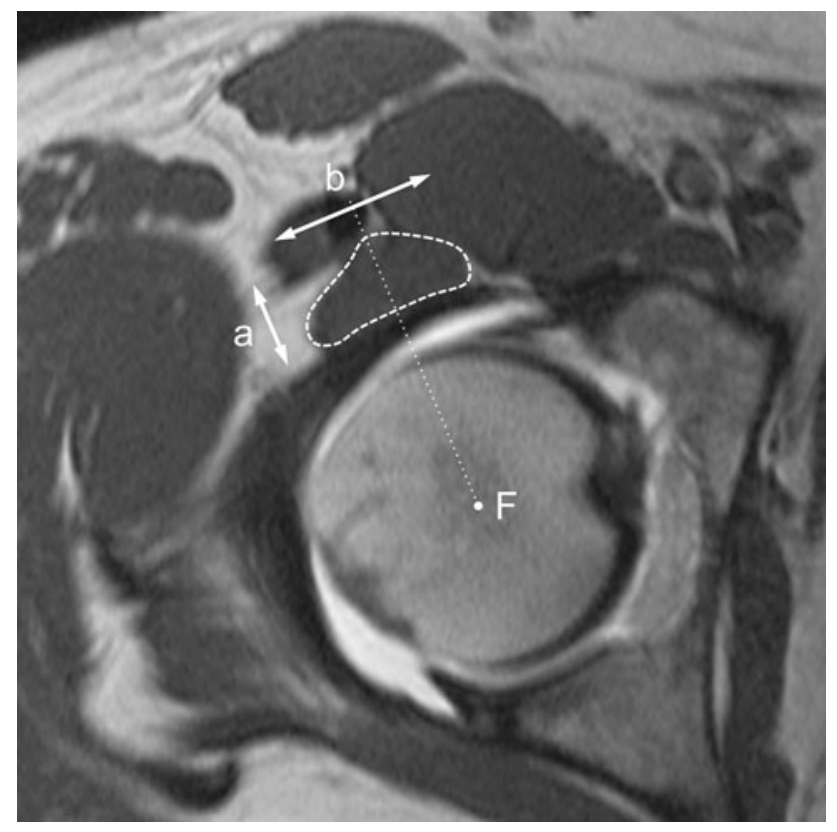

Fig. 1 An axial MR image shows the iliocapsularis muscle (dashed line) anterior of the hip. Five parameters were measured to describe morphologic features of the muscle: thickness (a), width (b), circumference (dashed line), cross-sectional area (dashed line), and fatty infiltration according to Goutallier et al. [11]. Muscle thickness (a) was measured along a radial line (dotted line) passing through the femoral head center $(\mathrm{F})$. The width (b) was measured perpendicular to the thickness (a).

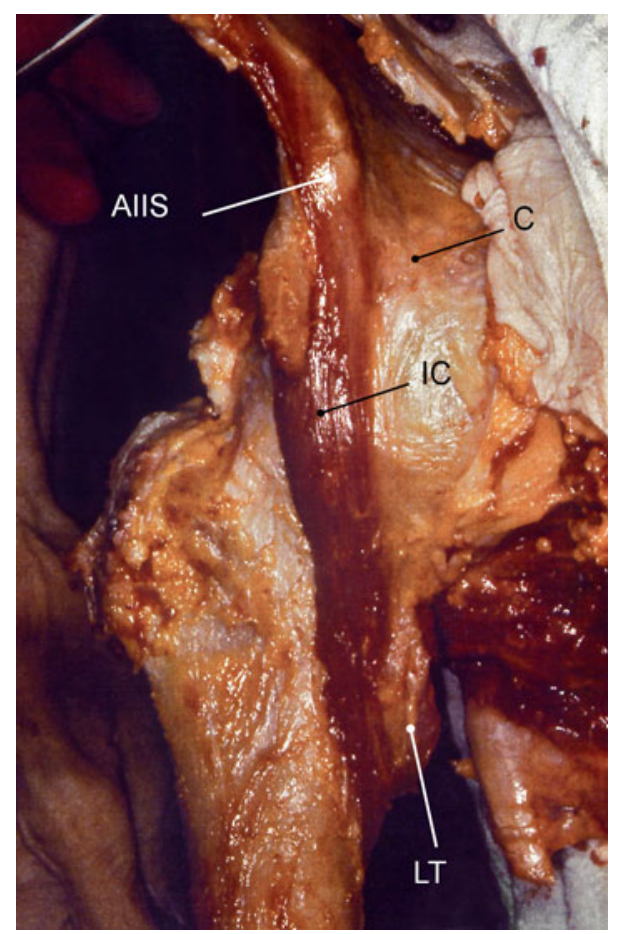

Fig. 2 The cadaver dissection shows the anterior aspect of the hip. The iliocapsularis muscle (IC) originates from the anterior-inferior iliac spine (AIIS) and the anterior hip capsule (C). Its insertion is located just distal to the lesser trochanter (LT). observations have not been confirmed and the true function of this muscle remains unknown.

The aims of this study were to quantify (1) the anatomic dimensions (thickness, width, circumference, CSA, and partial volume), and (2) the degree of fatty infiltration of the iliocapsularis muscle in hips with deficient and excessive acetabular coverage.

\section{Patients and Materials}

Using our digital institutional database, we identified 421 patients (480 hips) with developmental dysplasia of the hip (DDH) or excessive acetabular coverage (pincer type of femoroacetabular impingement) [10], who were seen at the outpatient clinic from November 1997 to October 2006 and who were younger than 50 years. In all cases after conventional radiography, a specific MR arthrography for the hip had been performed [18]. We excluded 42 patients (48 hips) with a history of known hip disorders (eg, LeggCalvé-Perthes disease), two patients (two hips) with muscle disorders (eg, muscle dystrophy), 79 patients (90 hips) with previous hip surgery, six patients (six hips) with skeletally immature hips (Stage 4 or less, according to Risser [20]), 12 patients (14 hips) with advanced osteoarthritis (Stage 2 or greater, according to Tönnis [27]), and 51 patients (58 hips) with incomplete or nondigital radiographic documentation. This left 229 patients (262 hips). We compared the morphologic features of the iliocapsularis muscle between two groups with different amounts of acetabular coverage: one with deficient coverage (DDH, Group I) and one with excessive coverage (Group II).

The allocation to either group was based solely on conventional AP pelvic radiographs as follows. DDH (Group I) was defined as a lateral center edge angle (LCE) less than $25^{\circ}$ [19] with a minimal acetabular index of $14^{\circ}$ [28], and classified according to Crowe et al. [2] (Table 1). Hips with an excessive acetabular roof (Group II) were defined as hips with a LCE angle exceeding $39^{\circ}$ [28] on the AP pelvic radiograph. One hundred forty-seven patients (177 hips) did not meet these radiographic criteria, leaving 82 patients ( 85 hips) for evaluation; 45 patients (45 hips) for Group I and 37 patients (40 hips) for Group II. The two groups were demographically similar (Table 1).

A standardized technique was used for the AP pelvic radiographs: film-focus distance $(1.2 \mathrm{~m})$, patient positioning (supine, legs $20^{\circ}$ internally rotated), and xray beam centering (midpoint between the symphysis and the line connecting the anterosuperior iliac spines) [25]. One observer (SDS) analyzed the individual acetabular morphologic features using validated computer software, $\mathrm{Hip}^{2}$ Norm (University of Bern, Switzerland) [24, 32]. The descriptive parameters were the LCE angle [30], acetabular 
Table 1. Demographic and radiographic data

\begin{tabular}{|c|c|c|c|}
\hline Parameter & Group I (dysplasia) & Group II (excessive coverage) & $\mathrm{p}$ Value \\
\hline Hips (number) & 45 & 40 & - \\
\hline Age of patients (years) & $34 \pm 9.7(17-49)$ & $33 \pm 11.0(17-49)$ & 0.69 \\
\hline Gender ( $\%$ male $)$ & 45 & 31 & 0.13 \\
\hline Right hips (\%) & 47 & 45 & 0.53 \\
\hline Weight (kg) & $71 \pm 16(48-110)$ & $69 \pm 11(52-93)$ & 0.66 \\
\hline Male & $80 \pm 12(65-101)$ & $77 \pm 8(67-93)$ & 0.42 \\
\hline Female & $66 \pm 16(48-110)$ & $64 \pm 9(52-85)$ & 0.54 \\
\hline Height (cm) & $171 \pm 9(157-188)$ & $171 \pm 9(159-192)$ & 1.00 \\
\hline Male & $178 \pm 8(165-188)$ & $179 \pm 7(170-192)$ & 0.70 \\
\hline Female & $167 \pm 6(157-176)$ & $167 \pm 6(159-182)$ & 0.96 \\
\hline BMI $\left(\mathrm{kg} / \mathrm{m}^{2}\right)$ & $25 \pm 5(18-37)$ & $23 \pm 4(18-32)$ & 0.11 \\
\hline Male & $25 \pm 4(19-32)$ & $24 \pm 4(20-30)$ & 0.31 \\
\hline Female & $25 \pm 5(18-37)$ & $23 \pm 4(18-32)$ & 0.22 \\
\hline \multicolumn{4}{|l|}{ Crowe classification [2] } \\
\hline Grade 1 & 43 & - & - \\
\hline Grade 2 & 2 & & \\
\hline Grade 3 & 0 & & \\
\hline Grade 4 & 0 & & \\
\hline LCE angle $\left(^{\circ}\right)[30]$ & $14 \pm 9(-16-24)$ & $45 \pm 5(39-59)$ & $<0.001$ \\
\hline Acetabular index $\left({ }^{\circ}\right)[28]$ & $21 \pm 6(14-38)$ & $-1 \pm 6(-13-14)$ & $<0.001$ \\
\hline Extrusion index (\%) [19] & $34 \pm 8(22-57)$ & $9 \pm 4(0-14)$ & $<0.001$ \\
\hline Femoral coverage $(\%)[24,25]$ & $64 \pm 12(32-87)$ & $92 \pm 6(79-100)$ & $<0.001$ \\
\hline
\end{tabular}

index [28], extrusion index [19], and femoral head coverage [24, 25] (Table 1).

MR arthrography was obtained according to a standardized technique [18]. In brief, the scans were performed using a Siemens Vision 1.5-T high field scanner (Erlangen, Germany) with a flexible surface coil after fluoroscopicguided intraarticular injection of saline-diluted gadoliniumDTPA (Dotarem 1:200, Guerbert AG, Paris, France). Transverse, sagittal, and coronal proton density-weighted and T1-weighted sequences were assessed. The axial slices used for measurements had a slice thickness of $4 \mathrm{~mm}$ and slice-to-slice distance of $4.8 \mathrm{~mm}$. Commercially available software, Osirix (Version 3.7, Geneva, Switzerland), was used for analysis [21]. The outline of the iliocapsularis muscle was defined manually on each MRI section from the origin of the muscle at the AIIS to the first section inferior to the femoral head. These margins were chosen because they represented the maximum available length of the muscle seen on the MR images for all patients.

We measured four parameters to describe the anatomic dimensions: thickness, width, circumference and CSA. Additionally, the degree of fatty infiltration of the muscle, according to Goutallier et al. [11], was assessed. All parameters were measured at two locations: $4 \mathrm{~cm}$ below the AIIS (Location A, according to Ward et al. [29]), and the first section inferior to the femoral head (Location B) (Fig. 3). One of us (DB) measured all parameters in the 95 hips. The muscle thickness was measured along a radial line passing through the femoral head center (Fig. 1). The width was measured perpendicular to the thickness (Fig. 1). Based on the manually defined outline of the iliocapsularis muscle, the circumference and the CSA were calculated automatically. Finally, the partial volume of the iliocapsularis muscle from its origin at the AIIS to Location B was calculated for each patient. All parameters were compared between the two groups, for all patients, and for men and women.

The reproducibility and reliability of these measurements were tested using a set of 25 randomly chosen hips. The blinded images were analyzed by two independent observers (DB, SDS) on two separate occasions at least 1 week apart. Intraobserver and interobserver variations in measuring the iliocapsularis dimensions and the classification of Goutallier et al. [11] were assessed using the intraclass correlation coefficients (ICC) and kappa value, respectively (Table 2 ).

A normal distribution was present for all continuous parameters, which were confirmed with the KolmogorovSmirnov test. Unpaired t-tests were used to compare the two study groups. Fisher's exact test was performed to 
Fig. 3A-B An (A) MR image and (B) three-dimensional drawing show the two locations for measurement of muscle morphology and fatty infiltration: $4 \mathrm{~cm}$ below the AIIS (Location A), and on the first section inferior to the femoral head (Location B)
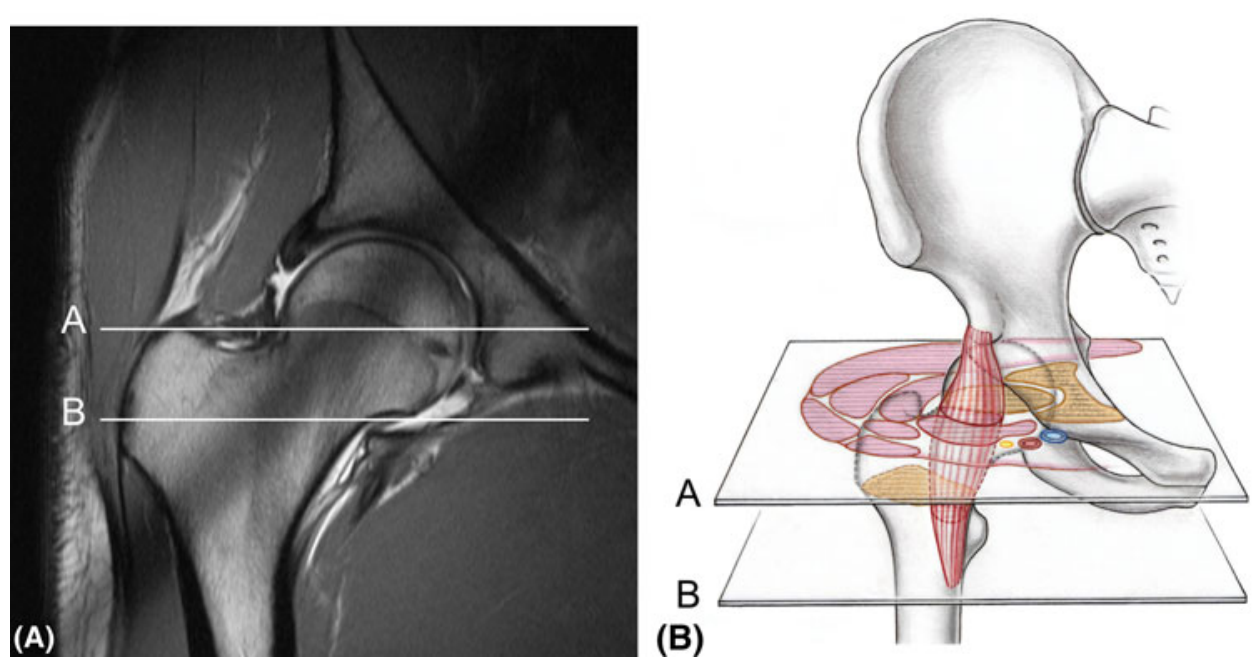

Table 2. Reliability and reproducibility of the evaluated study parameters

\begin{tabular}{llll}
\hline Parameters & ICC/Kappa intraobserver $1 *$ & ICC/Kappa intraobserver 2* & ICC/Kappa interobserver* \\
\hline Depth & $0.95(0.89-0.98)$ & $0.94(0.86-0.97)$ & $0.82(0.66-0.91)$ \\
Diameter & $0.90(0.78-0.95)$ & $0.93(0.85-0.97)$ & $0.80(0.66-0.90)$ \\
Circumference & $0.93(0.85-0.97)$ & $0.95(0.90-0.98)$ & $0.88(0.83-0.92)$ \\
Cross-sectional area & $0.94(0.87-0.97)$ & $0.92(0.82-0.97)$ & $0.81(0.69-0.90)$ \\
Classification of Goutallier et al. [11] & $0.85(0.70-1.00)$ & $0.80(0.61-0.98)$ & $0.79(0.72-0.87)$ \\
\hline
\end{tabular}

ICC $=$ intraclass correlation coefficient; * mean $(95 \%$ confidence interval).

assess associations between categorical variables. Assuming a mean normal muscle width of $2.15 \mathrm{~cm}$, an estimated standard deviation of $0.5 \mathrm{~cm}$, and a $20 \%$ muscular hypertrophy [3], we calculated a minimum sample size of 35 hips for each group to provide a level of alpha of 0.01 and a beta of 0.10 .

\section{Results}

We observed increased thickness, width, circumference, CSA, and partial volume of the iliocapsularis muscle in Group I compared with Group II (Table 3). Increased muscle dimensions were found in Group I for men and women separately, except for the muscular depth in men at Location A (Table 3).

Group I had a lower prevalence $(\mathrm{p}=0.04)$ of fatty infiltration compared with Group II at both locations (Table 3). There was no difference in terms of fatty infiltration for men between the two study groups for either location (Table 3). For women, there was a lower prevalence $(p=0.03)$ of fatty infiltration for Group $I$ at Location A (Table 3).

\section{Discussion}

The increased demand of a muscle or muscle groups causes hypertrophy of the corresponding muscular fibers [22] Conversely, decreased demand leads to muscular hypotrophy and fatty infiltration [31]. Based on the postulated function of a hip stabilizer [29], we questioned if dysplastic hips have (1) increased anatomic dimensions (thickness, width, circumference, CSA, and partial volume), and (2) less fatty infiltration of the iliocapsularis muscle, compared with hips with excessive coverage.

The first and main limitation was our inability to measure the muscle dimensions along its entire course, owing to the standard MRI protocol with a field of view optimized to detect intraarticular disorders [18]. The standard protocol does not necessarily include the distal portion of the iliocapsularis muscle. Therefore, the calculated muscle volume for the study patients (Table 3 ) is smaller than the total muscle volume. Second, we did not adjust the absolute dimensions of the iliocapsularis muscle to height or weight of the individual patient. However, a comparison of these absolute values is still eligible as the two study groups did not differ demographically (Table 1). Third, 


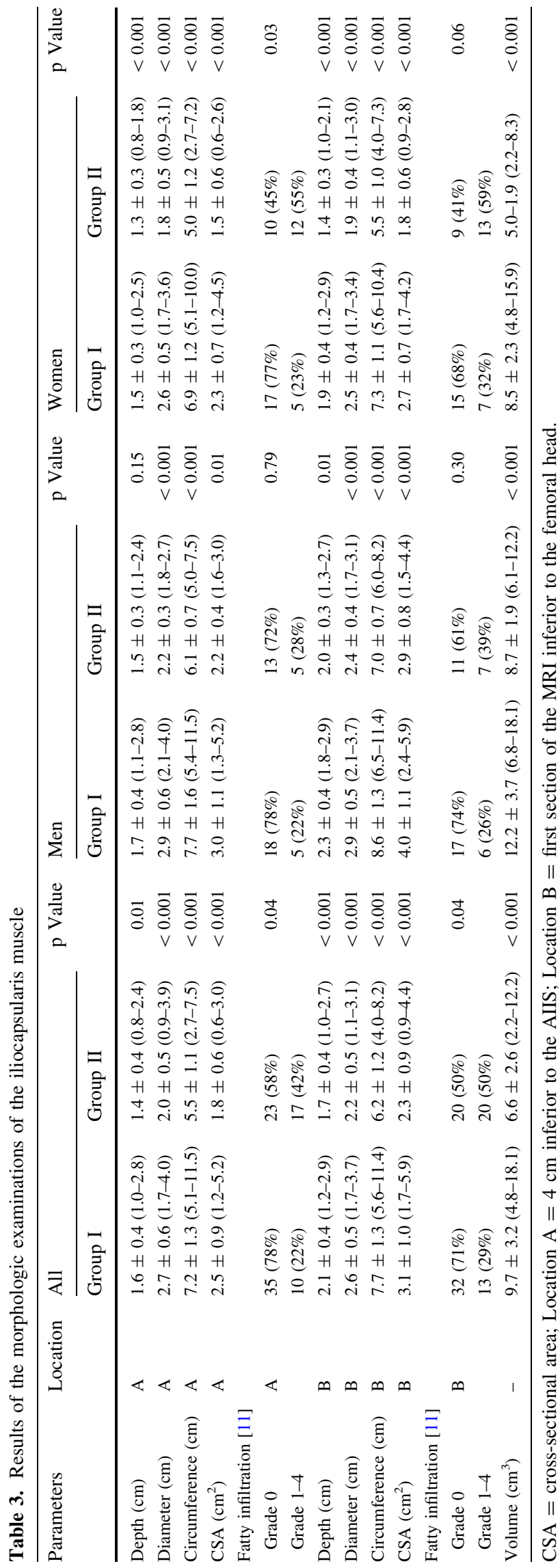

a false-positive fatty infiltration of the iliocapsularis muscle theoretically can result from intramuscular or perimuscular injection of the contrast agent if not applied correctly. However, this is rarely seen in our series and therefore should not jeopardize the conclusions.

The true function of the iliocapsularis muscle is unknown [29]. Based on the additional origin of the muscle on the hip capsule, it was postulated that it can tighten the anterior hip capsule [1, 26, 29]. Although, based on static concept, this should help stabilize the femoral head in dysplastic hips with deficient anterolateral acetabular coverage. Presumably the instability of the hip causes chronic overloads and shear forces, which are most pronounced in full extension and external rotation of the hip [17], motions where the iliocapsularis is maximally stretched. This would explain the muscle hypertrophy (Fig. 4). This hypertrophy may passively assist in constraining the femoral head in the deficient acetabulum. In hips with acetabular over-coverage, stabilization of the femoral head already is provided by the bony anatomy. A strong iliocapsularis muscle is superfluous. As shown in our study, the iliocapsularis muscle atrophies with fatty infiltration (Fig. 4).

Despite reports in the literature [14], the iliocapsularis muscle is a constant muscle and does not vary in human beings [29]; we found it in all patients. It also can be identified in primates [13], rats [5], reptiles [12], or birds [8]. We found only two reports describing the dimensions of the iliocapsularis muscle in human beings. Fetterolf [6] reported an approximate muscle width of $1 \mathrm{~cm}$, and Ward et al. [29] reported a thickness of $0.4 \mathrm{~cm}$ to $1 \mathrm{~cm}$ and a width of $1.8 \mathrm{~cm}$ to $2.5 \mathrm{~cm}$ at Location A. Although these numbers are similar to ours, a direct comparison is not possible owing to lack of complete descriptive statistics (mean, standard deviation). In primates, the width of the iliocapsularis ranges from $1 \mathrm{~mm}$ to $1.6 \mathrm{~cm}$, depending on the species [13].

We found only one study examining the structural variations and abnormalities of the iliocapsularis muscle. Kuhlman et al. [16] suggested that a hypertrophied iliocapsularis muscle would lead to excessive traction of the capsulolabral complex with subsequent labral disorders. Based on our observations, for several reasons it is more likely that those hips have symptomatic hip dysplasia rather than a primary disorder of the iliocapsularis muscle. First, the origin of the muscle is the AIIS and not the labral complex [29]. Therefore, detachment of the labrum by excessive traction of the iliocapsularis muscle is unlikely. Second, the hypertrophy of the iliocapsularis muscle is related to the deficient acetabular coverage and not a pathologic entity. Third, the observed labral tears in patients with DDH are related to increased joint contact pressure [23]. 
Fig. 4A-D (A) A drawing and an axial MR arthrography slice from a dysplastic hip (Group I, at $4 \mathrm{~cm}$ below the AIIS [Location A]) shows increased size (thickness, width, circumference, CSA) of the iliocapsularis muscle (IC) with decreased fatty infiltration compared with $(\mathbf{C})$ the drawing and (D) axial MR arthrography slice of the iliocapsularis muscle in a hip with excessive acetabular coverage (Group II). F = femoral head, $\mathrm{A}=$ acetabulum, $\mathrm{PA}=$ psoas major muscle, $\mathrm{PI}=$ psoas minor muscle, I = iliacus muscle, $\mathrm{R}=$ rectus femoris muscle, $\mathrm{S}=$ sartorius muscle, $\mathrm{TF}=$ tensor fascia muscle, $\mathrm{MI}=$ gluteus minimus muscle, $\mathrm{ME}=$ gluteus medius muscle, $\mathrm{MA}=$ gluteus maximus muscle, $\mathrm{O}=$ obturator internus muscle, $\mathrm{PE}=$ pectineus muscle, $\mathrm{A}=$ femoral artery, $\mathrm{V}=$ femoral vein, $\mathrm{N}=$ femoral nerve, $\mathrm{C}=$ hip capsule.

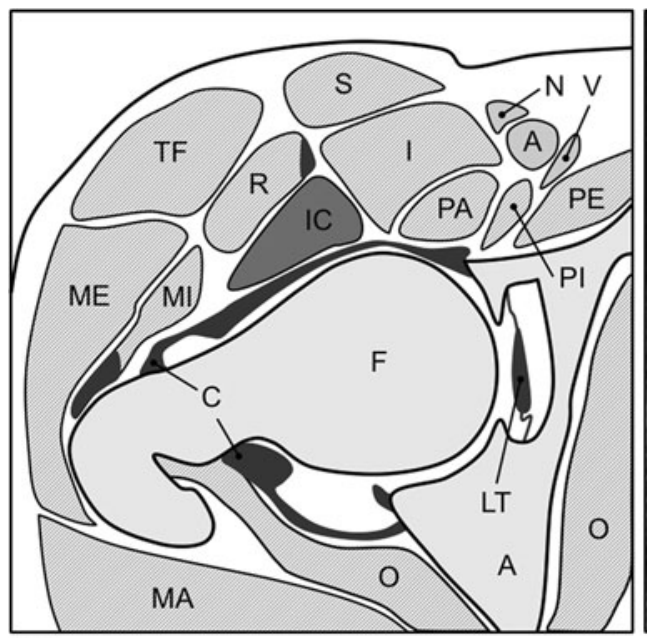

(A)

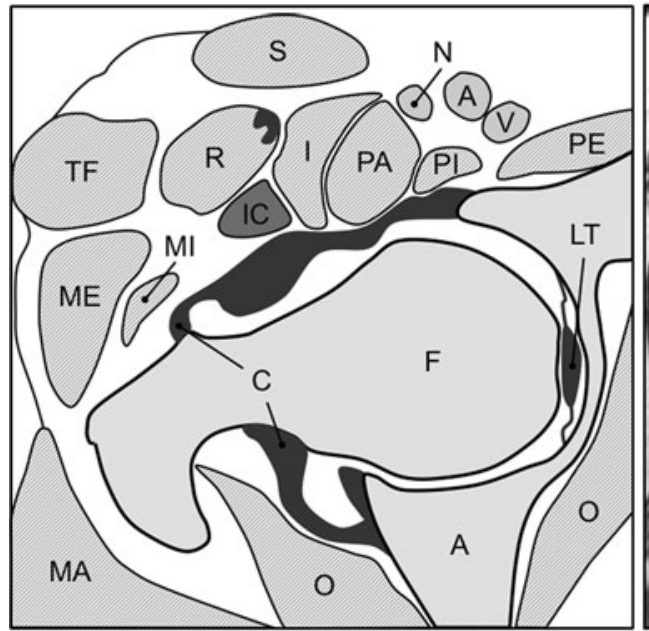

(C)

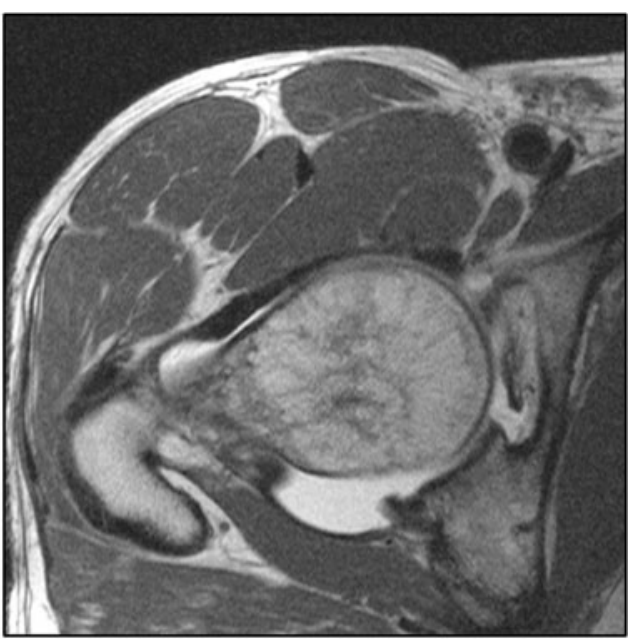

(B)

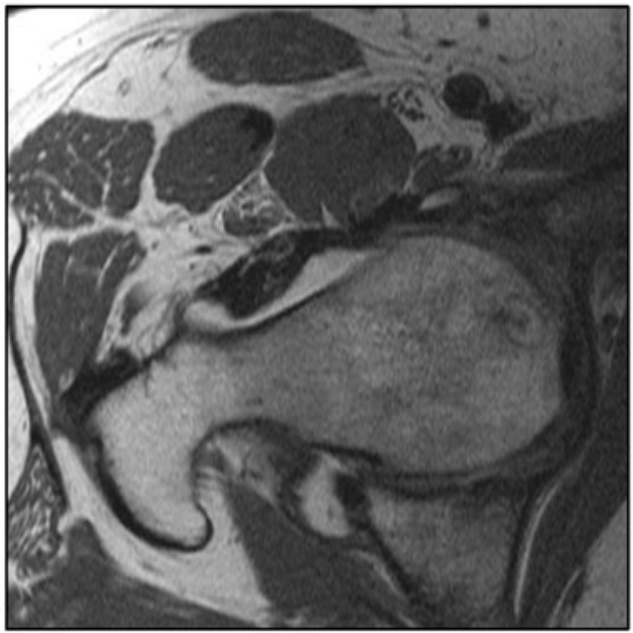

(D)
Although rarely mentioned, we believe the iliocapsularis muscle is clinically relevant as it serves as an important anatomic landmark in hip surgery. Its anteromedial border can be used to identify the joint capsule during periacetabular osteotomy using a modified Smith-Petersen approach [9]. It also is helpful during anterolateral or direct lateral approaches in identifying the iliopsoas tendon during tenotomy [4]. The anterolateral border of the iliocapsularis muscle is described as the ideal location for the capsulotomy in anterior THA [15]. Additionally, our observations can be used in daily clinical practice for decision making in patients with borderline cases of DDH $[7,28]$ or femoroacetabular impingement. Here the surgical strategy refers to the predominant underlying pathomechanism, which can be determined with the help of secondary morphologic alterations. Patients with a large iliocapsularis muscle without fatty infiltration most likely have acetabular dysplasia. These patients typically have a large labrum [17]. Improvement of the acetabular coverage, such as by periacetabular osteotomy [9], represents the causal therapy. Patients with an atrophied and fatty-infiltrated iliocapsularis muscle more likely have over-coverage of the acetabulum (pincer type of femoroacetabular impingement [25]). The labrum typically is small in these patients [17]. Reduction of the anterolateral acetabular coverage should be considered, either by trimming the excessive portion of the acetabular rim or reorientating the acetabulum with a periacetabular osteotomy.

The iliocapsularis muscle is a constant muscle whose size and quality vary depending on the morphologic features of the acetabulum. Our observations suggest it is important to stabilize the femoral head in a deficient acetabulum, where it typically is hypertrophied. In hips with excessive acetabular coverage, stabilization of the femoral head is provided by the bony anatomy. This leads to a decreased demand of the iliocapsularis muscle, followed by hypotrophy and fatty infiltration. MRI can be used to preoperatively evaluate the iliocapsularis muscle and to assist 
with treatment decisions in patients with hips having deficient or excessive acetabular coverage, and particularly in borderline cases.

Acknowledgment We thank Dr. Stefan Werlen from the Department of Radiology, Sonnenhof Clinic, Bern, Switzerland who provided the necessary MR data sets and provided expertise with MRI to perform the measurements.

\section{References}

1. Braus H. Spezielle Bewegungsapparate der unteren Extremität. In: Braus $\mathrm{H}$, ed. Anatomie des Menschen. 3rd ed. Berlin, Germany: Springer; pp. 420-508.

2. Crowe JF, Mani VJ, Ranawat CS. Total hip replacement in congenital dislocation and dysplasia of the hip. J Bone Joint Surg Am. 1979;61:15-23.

3. Cureton KJ, Collins MA, Hill DW, McElhannon FM Jr. Muscle hypertrophy in men and women. Med Sci Sports Exerc. 1988;20:338-344.

4. Dora C, Houweling M, Koch P, Sierra RJ. Iliopsoas impingement after total hip replacement: the results of non-operative management, tenotomy or acetabular revision. J Bone Joint Surg Br. 2007;89:1031-1035.

5. Eisler P. Die Homologie der Extremitäten. In: Brandes G, ed. Abhandlungen der Naturforschenden Gesellschaft zu Halle. Halle: Max Niemeyer; 1893-1895:87-344.

6. Fetterolf G. Variations in the muscles and nerves of the human body. University of Pennsylvania Medical Bulletin. Philadelphia, PA: University of Pennsylvania Press; 1910:174-180.

7. Fredensborg N. The CE angle of normal hips. Acta Orthop Scand. 1976;47:403-405.

8. Gadow H. Muskeln. In: Gadow H, ed. Zur vergleichenden Anatomie der Muskulatur der hinteren Gliedmasse der Ratiten. Jena, Germany: Gustav Fisher; 1880:12-55.

9. Ganz R, Klaue K, Vinh TS, Mast JW. A new periacetabular osteotomy for the treatment of hip dysplasias: technique and preliminary results. Clin Orthop Relat Res. 1988;232:26-36.

10. Ganz R, Parvizi J, Beck M, Leunig M, Nötzli H, Siebenrock KA. Femoroacetabular impingement: a cause for osteoarthritis of the hip. Clin Orthop Relat Res. 2003;417:112-120.

11. Goutallier D, Postel JM, Bernageau J, Lavau L, Voisin MC. Fatty muscle degeneration in cuff ruptures: pre- and postoperative evaluation by CT scan. Clin Orthop Relat Res. 1994;304:78-83.

12. Gregory WK, Camp L. Review and identification of muslces connected with the pelvis and sacrum in placentals, monotremes, sphenodon, and other reptiles, with inferred conditions in cynognathus. In: Lutz FE, ed. Bulletin of the American Museum of Natural History. New York, NY: The American Museum of Natural History; 1918:450-514

13. Hofer H, Schultz AH, Stark D. Primatologia. Handbuch der Primatenkunde. 4th ed. New York, NY: S. Karger; 1968.

14. Krause CFT. Varietäten der Muskeln. In: Krause W, ed. Handbuch der menschlichen Anatomie. Hannover, Germany: Hahn'sche Buchhandlung; 1880:87-120.
15. Kreuzer S, Matta JM. Single-incision anterior approach for total hip arthroplasty: Smith-Petersen approach. In: O’Connor MI, Griffin Ly, eds. Limited Incisions for Total Hip Arthroplasty. Rosemont, IL: American Academy of Orthopaedic Surgeons; 2007:1-14.

16. Kuhlman GS, Domb BG. Hip impingement: identifying and treating a common cause of hip pain. Am Fam Physician. 2009;80:1429-1434.

17. Leunig M, Podeszwa D, Beck M, Werlen S, Ganz R. Magnetic resonance arthrography of labral disorders in hips with dysplasia and impingement. Clin Orthop Relat Res. 2004;418: 74-80.

18. Leunig M, Werlen S, Ungersbock A, Ito K, Ganz R. Evaluation of the acetabular labrum by MR arthrography. J Bone Joint Surg Br. 1997;79:230-234.

19. Murphy SB, Ganz R, Müller ME. The prognosis in untreated dysplasia of the hip: a study of radiographic factors that predict the outcome. J Bone Joint Surg Am. 1995;77:985-989.

20. Risser JC. The iliac apophysis; an invaluable sign in the management of scoliosis. Clin Orthop. 1958;11:111-119.

21. Rosset A, Spadola L, Ratib O. OsiriX: an open-source software for navigating in multidimensional DICOM images. $J$ Digit Imaging. 2004;17:205-216.

22. Soares JM. Effects of training on muscle capillary pattern: intermittent vs continuous exercise. J Sports Med Phys Fitness. 1992;32:123-127.

23. Steppacher SD, Tannast M, Siebenrock KA. Labrumläsionen des Hüftgelenkes. In: Günther KP, Pohlemann T, eds. Orthopädie und Unfallchirurige up2date. Beckengürtel und untere Extremität. Stuttgart, Germany: Georg Thieme; 2008:215-232.

24. Tannast M, Mistry S, Steppacher SD, Reichenbach S, Langlotz F, Siebenrock KA, Zheng G. Radiographic analysis of femoroacetabular impingement with Hip2Norm-reliable and validated. J Orthop Res. 2008;26:1199-1205.

25. Tannast M, Siebenrock KA, Anderson SE. Femoroacetabular impingement: radiographic diagnosis-what the radiologist should know. AJR Am J Roentgenol. 2007;188:1540-1552.

26. Töndury G. Hüfte und Hüftgelenk. In: Töndury L, ed. Angewandte und Topographische Anatomie. 5th ed. Stuttgart, Germany: Georg-Thieme; 1981:462-478.

27. Tönnis D. General radiography of the hip joint. In: Tönnis D, ed. Congenital Dysplasia and Dislocation of the Hip. Heidelberg, Germany: Springer; 1987:100-142.

28. Tönnis D, Heinecke A. Acetabular and femoral anteversion: relationship with osteoarthritis of the hip. J Bone Joint Surg Am. 1999;81:1747-1770.

29. Ward WT, Fleisch ID, Ganz R. Anatomy of the iliocapsularis muscle: relevance to surgery of the hip. Clin Orthop Relat Res. 2000;374:278-285.

30. Wiberg G. The anatomy and roentgenographic appearance of a normal hip joint. Acta Chir Scand. 1939;83(suppl 58):7-38.

31. Williams MD, Ladermann A, Melis B, Barthelemy R, Walch G. Fatty infiltration of the supraspinatus: a reliability study. J Shoulder Elbow Surg. 2009;18:581-587.

32. Zheng G, Tannast M, Anderegg C, Siebenrock KA, Langlotz F. Hip2Norm: an object-oriented cross-platform program for $3 \mathrm{D}$ analysis of hip joint morphology using 2D pelvic radiographs. Comput Methods Programs Biomed. 2007;87:36-45. 